\title{
German Stroke School Vol. 3 auf dem neuroRAD 2021, 6.-8. Oktober 2021
}

Aufgrund der durchweg positiven Rückmeldungen zu den zwei bisher stattgefundenen German Stroke Schools in den Jahren 2018 und 2019 und der großen Nachfrage nach diesem Format können wir Ihnen nun für das Jahr 2021 wieder eine Corona-konforme Version der German Stroke School anbieten, das spezielle Vortrags- und Simulationsformat zur interventionellen Schlaganfallbehandlung der Deutschen Gesellschaft für Neuroradiologie (DGNR).

Während die Lectures im Rahmen des Online-Kongresses diesmal virtuell stattfinden werden (6.10.2021), können wir Ihnen für den praktischen Teil einen 1-tägigen Intensivkurs in München (12.10.2021) bzw. Dresden (14.10.2021) anbieten.

Trainiert wird in Kleingruppen an modernsten Angiografie-Simulatoren (Mentice) und an Flussmodellen im Katheterlabor. Hierbei können die Teilnehmer/Teilnehmerinnen echte Materialien und Devices sowie deren korrekte Anwendung kennenlernen.

Ziel ist die selbstständige Durchführung der zerebralen Thrombektomie an Patienten/ Patientinnen im Anschluss an den Kurs. Vorkenntnisse in der (zerebralen) Angiografie sind für einen größtmöglichen Benefit empfehlenswert.

Nehmen Sie die Gelegenheit wahr, aktuelle
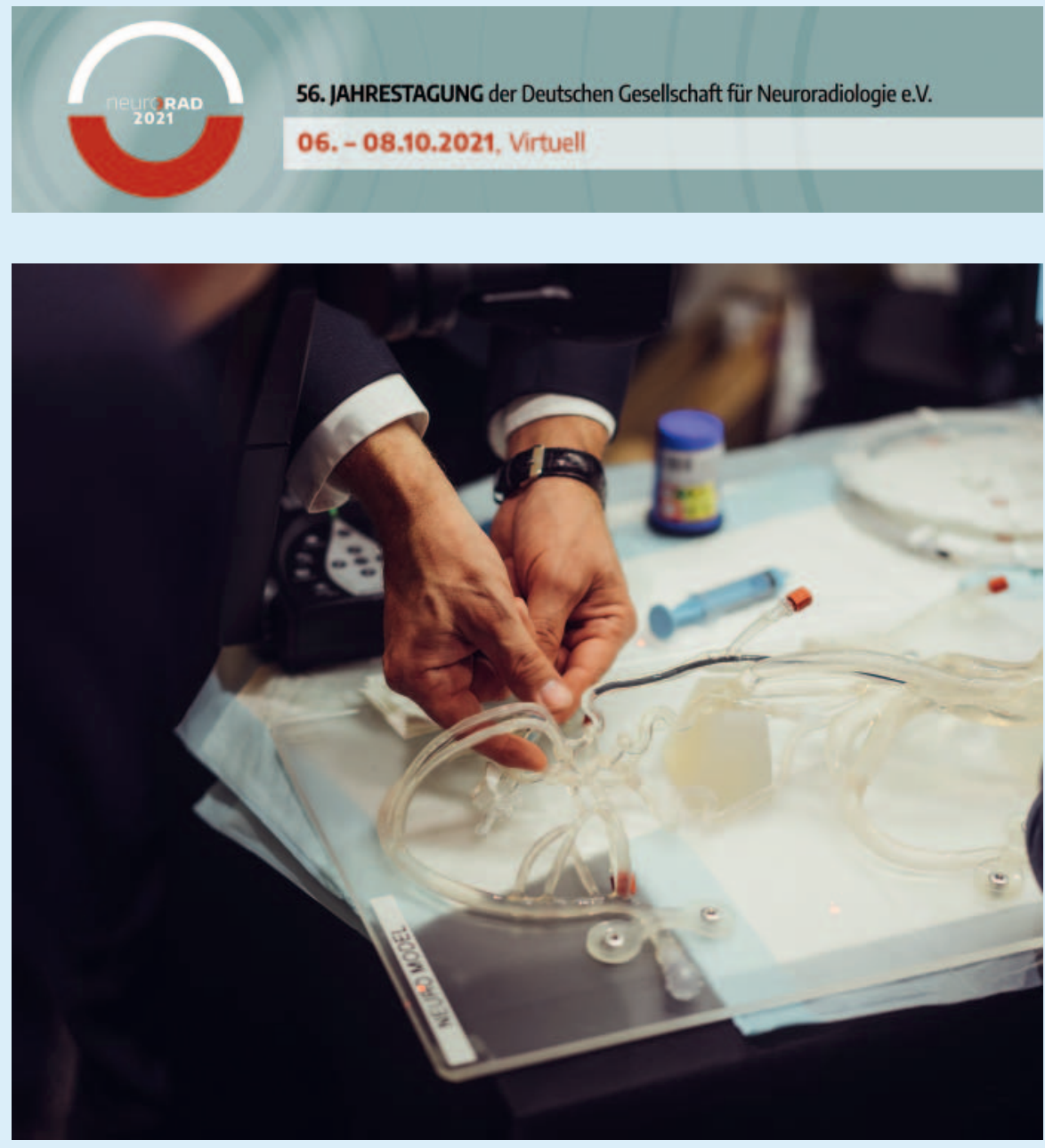

Echte Materialien anfassen, ausprobieren, mit Experten/Expertinnen diskutieren - ohne Einflussnahme der Industrie. (copyright: Christian Maegerlein).

Techniken, spezielle Tricks und Strategien von ausgewiesenen Experten/Expertinnen zu erlernen! 
DIE FAKTEN:

\section{GSS-VORLESUNGSPROGRAMM am 6. Oktober 2021}

Umfassendes Vortrags-Curriculum mit allen relevanten Themen wie Schlaganfall-Pathophysiologie, Update zu aktuellen Erkenntnissen und offene Fragen in der interventionellen Schlaganfallbehandlung, erweiterte Schlaganfallbildgebung zur Auswahl von Patienten/Patientinnen, interventionelle Behandlungstechniken, Optimierung von Arbeitsabläufen und Management von Komplikationen.

Die Vorträge sind für alle Teilnehmer/Teilnehmerinnen des neuroRAD 2021 kostenfrei!

\section{FULL-DAY-HANDS-ON-TRAINING in München (12.10.2021) und in Dresden (14.10.2021)}

Ein ganzer Tag Hands-on-Training in München oder Dresden, mit den neuesten Simulationstechniken und unter Anleitung von erfahrenen Tutoren/Tutorinnen. Dieser Teil der GSS richtet sich besonders an junge Radiologen/Radiologinnen und Neuroradiologen/Neuroradiologinnen mit angiografischer Erfahrung.

STRENG LIMITIERT AUF 32 TEILNEHMER/TEILNEHMERINNEN, MUSS SEPARAT GEBUCHT WERDEN!

Kurssprache: Die Vorlesungen sind in Englisch, Hands-on-Teile werden in Deutsch und Englisch angeboten.

Jetzt anmelden auf www.neurorad.de.

>> Thrombektomie mit Simulatoren der neuesten Generation. Max. 2 Personen pro Simulator und persönliche Betreuung garantieren einen maximalen Lernerfolg!

\section{KOMMENTARE VON TEILNEHMERN/TEILNEHMERINNEN DER GSS 2019}

„[...] insbesondere auch die praktischen Übungen am Simulator und den Flussmodellen waren extrem hilfreich. Nach dem Kurs fühle ich mich bereit für „echte“ Schlaganfallbehandlungen.“

„[...] echte Insider-Tipps direkt von den Profis zu bekommen, das war wirklich großartig. Was ich da gelernt habe, kann man in keinem Buch lesen."

„Besonders spannend fand ich auch die Fallbesprechungen. Die Experten haben uns auch gezeigt, wo Gefahren und Probleme lauern. Ich bin dankbar, dass auch mögliche Komplikationen und Probleme angesprochen wurden."

„Die German Stroke School würde ich definitiv jedem empfehlen, der zeitnah selbstständig thrombektomieren möchte!“ 